Revista de Psicología Vol. 29 (2), 2011 (ISSN 0254-9247)

\title{
Latitud sur y control económico del hogar por la mujer peruana
}

\author{
Federico R. León ${ }^{12}$ \\ León \& Bustamante Consultores, Lima, Perú
}

\begin{abstract}
Mayor autonomía de la mujer sureńa versus mayor sometimiento tradicional al marido por la norteña fueron conjeturados en 1984 para explicar variaciones en el deseo fecundatorio de las mujeres peruanas. Un análisis de datos de la Encuesta Demográfica y de Salud Familiar Continua Perú 2004-2008 apoya esta hipótesis al revelar un crecimiento significativo de norte a sur del control de la mujer sobre el ingreso del cónyuge y, menos consistentemente, las decisiones adquisitivas hogareńas. Estas relaciones no son explicadas por variables correlacionadas, como la etnicidad indígena, poder material/informacional de la mujer, diferencia de edad con el marido, o que trabaje por dinero. Los hallazgos sugieren nuevas hipótesis, sobre la distribución de la asertividad y la calidez en el territorio peruano.

Palabras clave: norte sur, mujer peruana, decisiones económicas del hogar, asertividad.
\end{abstract}

\section{South latitude and household economic control by Peruvian women}

Southern women's greater autonomy versus northern women's more traditional submission to the husband were hypothesized in 1984 to explain variations in Peruvian women's fertility desires. An analysis of data from Peru 2004-2008 Continuous Demographic and Family Health Survey supports this hypothesis by showing a significant north-to-south growth of women's control upon husband's income and, less consistently, household purchasing decisions. These relationships are not explained by variables also correlated with meridionality, such as aboriginal ethnicity, women's material/informational power, age difference with the husband's, or working for cash. Findings suggest new hypotheses, concerning the distribution of assertiveness and warmth in the Peruvian territory.

Keywords: North South, Peruvian woman, household economics decisions, assertivity.

1 El autor agradece las sugerencias de Nina Bustamante de León.

2 PhD y Postdoctorado en Psicología. Investigador y consultor en comportamiento laboral, drogas, conducta reproductiva/ servicios de salud en el Perú y en el extranjero. Fundador de CEDRO y miembro del Americas Regional Advisory Panel, World Health Organization. Contacto: Av. Javier Prado 7427, Lima 3, Perú; federicorleone@gmail.com; www.leonybustamante.com. 

Este artículo aborda una hipótesis geográfico-psicosocial que se formuló hace más de un cuarto de siglo y permanecía sin verificar; la hipótesis explicaría el eje fecundatorio norte-sur del Perú y el eje nortesur de uso de sustancias psicoactivas. El primero se refiere al deseo de tener hijos de las mujeres (Morales, 1983) y su conocimiento de métodos anticonceptivos (Alcántara, 1983), que crecen de sur a norte. En un análisis de la base de datos de la Encuesta Nacional de Prevalencia de Anticonceptivos (INEI, 1983), León (1984) verificó la presencia del eje norte-sur del deseo de hijos tanto en la costa como en la sierra peruanas manteniendo constantes el tamaño de la familia, la educación y el trabajo de la mujer. Para explicarlo, conjeturó que la mujer del sur se distinguía por un afán mayor de independencia y desarrollo personal, mientras que la del norte estaría más gobernada por expectativas tradicionales de rol, según las cuales corresponde a la mujer dedicarse a la reproducción y el cuidado de los niños. Es decir, la condición de la mujer del norte sería más deficitaria en términos de empoderamiento (Malhotra, Schuler \& Boender, 2002).

El componente psicosocial de esta hipótesis es consistente con los resultados de estudios subsecuentes en diversas partes del mundo que han demostrado una asociación importante entre el grado de autonomía de las mujeres y sus actitudes y comportamientos reproductivos. Al respecto, se puede consultar la revisión de la literatura de Blanc (2001) y los estudios posteriores de Al Riyami, Afifi y Mabry (2004); Casique (2003); Chavoshi, Abbasi-Shavasi y McDonald (2004); Feldman, Zaslavsky, Ezzati, Peterson y Mitchell (2009); Jejeebhoy (2002); Kishor y Gupta (2009); León (2011); Moursund y Kravdal (2003); Mumtaz, Slaymaker y Salway (2005); Roy y Niranjan (2004); Saleem y Bobak (2005); Sathar, Callum y Jejeebhoy (2001); y, Woldemicael (2009).

El segundo eje concierne a la prevalencia de vida y uso actual de alcohol, tabaco y hoja de coca, que crecen en sentido opuesto, es decir, 
de norte a sur, con excepción del uso actual de alcohol en la costa, según análisis efectuados por León (1987) en las tablas de resultados de Drug use and abuse in Peru: An epidemiological investigation of drugs in urban Peru (Jutkowitz et al., 1986). Para reconciliar con la interpretación geográfico-psicosocial su hallazgo sobre sustancias psicoactivas, León (1987) propuso una explicación según la cual las madres más independientes, entretenidas en aspiraciones de desarrollo personal, causarían en sus hijos ciertas carencias afectivas que se expresarían luego, en su juventud y adultez, en un mayor consumo de alcohol, tabaco y/u hoja de coca. Fundamentó esta interpretación citando trabajos sobre predictores del uso de drogas en la juventud que señalaban la importancia de factores relativos a antiguas interacciones padres-hijos: la falta de cercanía en la relación y la falta de involucramiento materno en las actividades de los hijos (Baumrind, 1985; Blum, Henry \& Sanford, 1972; Braucht, Brakarsh, Follingstad \& Berry, 1973; Brook, Linkoff \& Whiteman, 1980; Kandel, 1982; Kandel, Kessler \& Margulies, 1978; Kaplan, 1982; Kim, 1979; Penning \& Barnes, 1982). Así se entendería el mayor uso de sustancias psicoactivas observado a medida que el poblador peruano se aleja de la línea ecuatorial.

La hipótesis explicativa no ha estimulado investigaciones: la literatura no registra estudios sobre la distribución geográfica de la autonomía de la mujer en el Perú pese a que la demostración de validez de la hipótesis enriquecería sustancialmente el conocimiento de la realidad nacional. Una oportunidad de ponerla a prueba se presentó recientemente gracias a la inclusión de variables clave en la Encuesta Continua de Demografía y Salud Familiar Perú 2004-2008 conducida por el INEI (Instituto Nacional de Estadística e Informática) y ORC Macro. La encuesta incluyó un juego de ítems sobre el papel de la mujer en la toma de decisiones económicas del hogar que faculta al investigador a distinguir su autonomía (toma de decisiones por su cuenta) de su participación en las decisiones con el marido (decisiones conjuntas) y su exclusión de las decisiones (desempoderamiento). También introdujo mediciones GPS (Global Positioning System), de las cuales la más importante es la coordenada de latitud, que ofrece información exacta 
sobre la distancia entre el conglomerado tocado por la encuesta y la línea ecuatorial, una medición mucho más precisa que la tricotomía norte-centro-sur usada por León $(1984,1986,1987)$. Facilita además la prueba de la hipótesis al interior de la selva, región natural omitida en sus estudios iniciales porque la variable geográfica estaba definida por las regiones de planificación de entonces en la base de datos del Instituto Nacional de Estadística (norte, centro, sur, oriente).

El objetivo específico de la investigación que sigue fue determinar si el control ejercido por la mujer peruana en la toma de decisiones económicas del hogar es contingente al grado de latitud sur de su hábitat. El estudio se diseñó tomando en cuenta posibles efectos provenientes de variables conocidas por su relación con la meridionalidad (e. g. el quechua y el aimara son más hablados en el sur que en el norte del país) o que podrían estar relacionadas con la meridionalidad.

\section{Método}

\section{Datos}

La Encuesta Continua Perú 2004-2008 (DHS, 2010) fue parte del emprendimiento internacional Demographic and Health Surveys, cuya metodología estándar a través de países entraña un diseño de muestra multi-etápico y estratificado, y ponderaciones especialmente calculadas para proporcionar estimados de las variables de interés con representatividad a nivel nacional y sub-nacional. Las etapas fueron de selección de conglomerados y de hogares dentro de ellos, la estratificación fue urbano-rural y la representatividad alcanzó el nivel del departamento. Las mujeres elegibles dentro de las viviendas fueron todas aquellas en edad fértil, es decir, de 15 a 49 años de edad. La encuesta tuvo lugar a lo largo de cinco años, desde octubre de 2003 hasta noviembre de 2008. Se diseñó para superar la falta de información entre las encuestas DHS que se realizaban cada cinco años en el Perú. Para alcanzar este objetivo, el tamaño de muestra buscado (33000 hogares) fue dividido en cinco 
partes anuales correspondientes aproximadamente a 6600 hogares por ańo. La muestra fue diseñada de manera que cada año fuese representativa a nivel nacional y al final del quinto año lo fuera también a nivel departamental. Sin embargo, como resultado de una decisión gubernamental de crear una línea de base de indicadores representativos al nivel del departamento, la muestra del año 2008 fue aumentada considerablemente.

Los datos de la muestra ampliada carecen de información GPS y no fueron tenidos en cuenta en el estudio reportado en este artículo. Nótese también que la base de datos usada en el presente estudio es distinta de aquella que el INEI y ORC Macro (2010) usaron para su reciente informe Perú: Encuesta Demográfica y de Salud Familiar ENDES Continua, 2009.

La base de datos usada es del tipo Individual Recode, cuya unidad de análisis es la mujer individual y cuyos códigos de respuesta para la mayor parte de las variables están estandarizados a través de los países (otras variables incluidas son de interés particular de cada país.) Se integró al Individual Recode la base de datos GPS, cuya unidad de análisis es el conglomerado $(N=1409)$. El análisis se efectuó después de excluir a las solteras, viudas o divorciadas. La medición de la participación de la mujer en las decisiones económicas del hogar es relativa al rol del marido y, por ende, se aplica solo al caso de las casadas o informalmente unidas. De allí que también fueran excluidas las mujeres cuyos esposos o compañeros no vivían con ellas. Hubo 17862 casos que satisfacían los criterios de inclusión.

\section{Mediciones}

La encuesta abordó temas que permitieron la medición de una variable independiente de métrica continua (latitud), una variable dependiente multinomial (decisiones autónomas de la mujer, conjuntas con el marido, desempoderamiento total de la mujer), dos variables de segmentación (ciudad/pueblo/campo y costa/sierra/selva), y 12 variables sociodemográficas continuas o dicotómicas de control. 
- Latitud: la información proporcionada por el GPS está en grados decimales, donde los decimales reemplazan a los minutos y segundos de la medición geográfica estándar. Todos los casos individuales incluidos en un conglomerado recibieron la misma medición de latitud. Para facilitar posibles comparaciones futuras con hábitats al norte de la linea ecuatorial, se mantuvo en el análisis el signo negativo que corresponde a la latitud sur. Se eliminó un caso con más de $-9^{\circ}$ de latitud que había sido erróneamente asignado al departamento de Amazonas.

- Tomador de decisiones: DHS preguntó, ¿Quién toma generalmente las siguientes decisiones, principalmente usted, su esposo/ compañero, usted y su esposo/compañero conjuntamente, alguna otra persona, o usted y alguna otra persona conjuntamente? Los ítems económicos fueron tres: Hacer compras grandes del hogar, Hacer compras para necesidades diarias del hogar, y Cómo se gasta(ba) el dinero que su esposo/compañero gana(ba). Respecto a cada ítem, Esposo/compañero y Alguna otra persona (mujer desempoderada) fueron distinguidos de La entrevistada y su esposo/compañero y La entrevistada y alguna otra persona (decisión conjunta), y de La entrevistada (autonomía de la mujer). Se constató que la participación de personas ajenas a la pareja en las decisiones económicas del hogar es mínima en el Perú, donde la familia nuclear es virtualmente universal. Pese a que se retuvo la categorización, justifica hablar de aquí para adelante de decisiones del marido, y no del marido y alguna otra persona, en casos de desempoderamiento de la mujer, y del marido y la mujer, no de los dos y alguna otra persona, en casos de decisión conjunta.

- Tipo de lugar de residencia: es el lugar donde la mujer fue encuestada. DHS lo codificó 1 = ciudad capital y cualquier ciudad con más de 1 millón de habitantes; 2 = ciudad con más de 50000 habitantes; $3=$ pueblo, es decir, cualquier otra área urbana; y 4 = área rural o campo. Como la ciudad de Lima fue la única en la primera categoría, se combinó los códigos 1 y 2 en uno solo.

- Región natural de residencia: Pulgar Vidal (1938) distinguió una región chala o costa que se extiende desde el océano Pacífico hasta los 500 m.s.n.m. y una región yunga marítima que alcanza los 2500 
m.s.n.m. en los contrafuertes occidentales de los Andes. Ambas fueron comprendidas en la definición de Resto Costa de DHS, que distinguió a esta región de Lima Metropolitana; aquí se les reunió dentro de la categoría Costa. La sierra comprendió las regiones naturales quechua, suni, puna y janca (Pulgar Vidal, 1938), es decir, el territorio comprendido desde los 2500 m.s.n.m. por el lado occidental de los Andes hasta su máxima altura y desde allí hasta los 2500 m.s.n.m. por el lado oriental, incluyendo los valles o las quebradas interandinos que caen por debajo de los 2500 m.s.n.m. La selva incluyó las regiones rupa rupa o selva alta (entre los 500 y los 2500 m.s.n.m.) y omagua o selva baja (83 a 500 m.s.n.m.) de la Amazonía peruana.

Nueve casos encuestados a 2875 m.s.n.m. y 26 por encima de los 4000 m.s.n.m., erróneamente asignados a Costa en la base de datos, fueron reasignados a Sierra. Veinticinco casos encuestados a 420 m.s.n.m., $-7.2^{\circ}$ de latitud, y $-79.1^{\circ}$ de longitud fueron reasignados de Sierra a Costa. Veinte casos a 1311 m.s.n.m., $-12.9^{\circ}$ de latitud y $-73.6^{\circ}$ de longitud fueron reasignados de Sierra a Selva. Lo mismo se hizo con 34 casos a 1814 o 2070 m.s.n.m. encuestados en latitud $-10.6^{\circ}$ y longitud $-75.4^{\circ}$ y con 54 casos del departamento de San Martín encuestados a alturas por debajo de los 2500 m.s.n.m. Estas correcciones abarcan a menos del uno por ciento de los casos (0,95\%).

- Puntaje factorial de riqueza: DHS proporciona un índice de riqueza del hogar construido a base de características de la vivienda (material del piso y el techo, fuente de agua, etc.) y activos (propiedad de radio, refrigeradora, bicicleta, moto, etc.). A cada variable le asigna un peso (puntaje factorial) generado mediante el análisis de componentes principales y combina y estandariza los valores resultantes con relación a una distribución normal con un promedio de 0 y una desviación estándar de 1 .

- Oriunda del lugar: DHS preguntó a la mujer cuánto tiempo había vivido continuamente en el lugar de la encuesta. Las respuestas fueron recodificadas Siempre $=1$ y No siempre $=0$.

- Lengua materna española: DHS preguntó a la mujer, ¿Cuál es el idioma o lengua materna que aprendió en su niñez? Las respuestas, codificadas originalmente como 1 = castellano, 2 = quechua, $3=$ 
aimara, 4 = otro indígena, y 5 = extranjero, fueron recodificadas como $1=$ castellano y $0=$ cualquier otro. El código 5 representó menos del $0.00 \%$ de los casos.

- Edad en años completados: la edad de la mujer fue calculada considerando su fecha de nacimiento y la fecha de la entrevista. DHS formuló dos preguntas (¿En qué mes y año nació usted? y ¿Qué edad alcanzó en su último cumpleaños?) y corrigió las inconsistencias.

- Diferencia con la edad del marido: DHS le preguntó a la mujer cuál era la edad del marido. En el análisis de datos se restó la edad de la mujer de la edad del marido.

- Educación en años simple: DHS preguntó, ¿Alguna vez asistió a la escuela? y ¿Cuál fue el año o grado más alto de estudios que aprobó (implicando también la instrucción universitaria)?, y calculó el número total de ańos requeridos para alcanzar el grado máximo de estudios indicado por la mujer.

- Alfabetismo: DHS asignó a priori el puntaje 2 a las mujeres con instrucción secundaria o universitaria. A las que tenían algún grado de instrucción primaria les dio una tarea de lectura y asignó el puntaje 0 a quienes no podían leerlo, 1 a quienes podían leer parte de la oración, y 2 a quienes la leían completa.

- Número de hijos vivos: esta es la suma que hizo DHS de respuestas a preguntas sobre el número total de hijos residentes en la vivienda, hijos residentes fuera de la vivienda, hijas residentes en la vivienda, e hijas residentes fuera de la vivienda.

- Trabajo por dinero: se ha encontrado consistentemente a través de países que es el trabajo por dinero, no por pago en especie, lo que confiere poder doméstico a la mujer (Kishor \& Subaiya, 2008). DHS preguntó a la mujer si había trabajado en los últimos 12 meses y si había recibido dinero por su trabajo, se le había pagado en especie, una combinación de dinero y especie, o no se le había pagado. Las dos variables fueron combinadas, asignándose 0 a la mujer que no había trabajado en los últimos 12 meses, o lo había hecho pero recibiendo solo paga en especie, y 1 a quien había trabajado y recibido su paga en dinero y especie o solo en dinero. 
- Escucha de radio: DHS preguntó a la mujer si escuchaba radio y con qué frecuencia: nunca (0), menos de una vez a la semana (1), por lo menos una vez a la semana (2), o casi todos los días (3).

- Visión de TV: la pregunta y la codificación de esta variable son similares a las de la anterior.

- Lectura de periódicos: ídem.

\section{Estrategia analitica}

A causa de ciertas consideraciones metodológicas y prácticas, las bases de datos DHS sub-representan o sobre-representan poblaciones específicas. Por ejemplo, en el presente caso, el tamaño de muestra de Lima Metropolitana está muy por debajo de lo que le correspondería por su magnitud demográfica porque es suficiente para alcanzar estimados con un error estándar aceptable, mientras que el del departamento de Madre de Dios lo excede ampliamente; si correspondiera a su realidad demográfica, sería demasiado pequeño y no permitiría obtener estimados precisos a nivel departamental. La estratificación urbanorural agrega complejidad. Las ponderaciones (pesos) por vivienda que vienen con las bases de datos DHS pueden ser usadas para restablecer el balance cuando se trata de obtener un porcentaje, un promedio o una mediana que sea representativo/a. Estas ponderaciones, sin embargo, no son aplicables para estimar relaciones (Rutstein \& Rojas, 2006), es decir, no se les puede usar en el cálculo de correlaciones o regresiones. Dada esta situación, el estudio empleó dos enfoques analíticos:

- Departamentos extremos. Contribuiría a reforzar la credibilidad de la hipótesis explicativa de León (1984) que en el departamento más meridional de la costa peruana (intacto y con ponderaciones) se encuentre mayor control de las decisiones económicas del hogar por parte de la mujer que en el más septentrional (intacto y con ponderaciones), y que lo mismo se observe en sierra y selva.

- Relaciones entre variables. Para evaluar relaciones entre latitud y control de las decisiones por parte de la mujer comprendiendo todo el país y manteniendo constantes las variables que podrían confundir 
los resultados, se optó por minimizar los sesgos de representatividad usando como variables de segmentación la región natural cruzada con el tipo de residencia y utilizando como variables de control las mediciones sociodemográficas consideradas en el estudio. De esta manera, los sesgos de representatividad se debilitarían como hipótesis alternativas para dar cuenta de los resultados. Así, si emergiese una relación significativa y de la misma dirección entre latitud y control de las decisiones en cada una de las nueve sub-muestras (tres regiones x tres lugares de residencia), difícilmente la podría explicar algún sesgo específico.

A fin de hacer más fácilmente comparables los impactos de las variables sociodemográficas, de métrica original desigual, se optó por estandarizarlas antes de calcular las regresiones, produciendo valores $z$ a nivel de la muestra global $(N=17861)$. De esta manera, los coeficientes exponenciales de regresión (odds ratios) asociados a ellas indicarían la medida en que un aumento de una desviación estándar en el predictor, en comparación al puntaje promedio, se asocia con un aumento en la probabilidad de que ocurran decisiones conjuntas o autónomas de la mujer versus su exclusión de las decisiones. La correlación entre estas variables y la latitud dio lugar a la selección de las más consistentemente vinculadas al grado de meridionalidad, las cuales fueron sometidas a análisis de consistencia interna (Cronbach, 1951) para formar escalas y reducir así el número de variables. El modelo estadístico finalmente empleado para cada área decisional fue la regresión logística multinomial, es decir, una regresión logística cuya variable dependiente tiene más de dos categorías, una de las cuales es usada como referencia.

En este estudio se usó como referencia el desempoderamiento de la mujer para evaluar los efectos de las variables independientes sobre la toma de decisiones conjunta o autónoma de ella. Los predictores incluyeron el grado de latitud y las variables sociodemográficas seleccionadas y sintetizadas, agregándose cada efecto después de ajustar todos los otros (SPSS, 2008). 


\section{Resultados}

\section{Departamentos extremos}

En las figuras 1, 2, y 3 las proporciones han sido ponderadas, el número de casos entre paréntesis representa el tamaño de muestra ponderado, M. D. significa Madre de Dios, y los datos se refieren a los departamentos intactos.

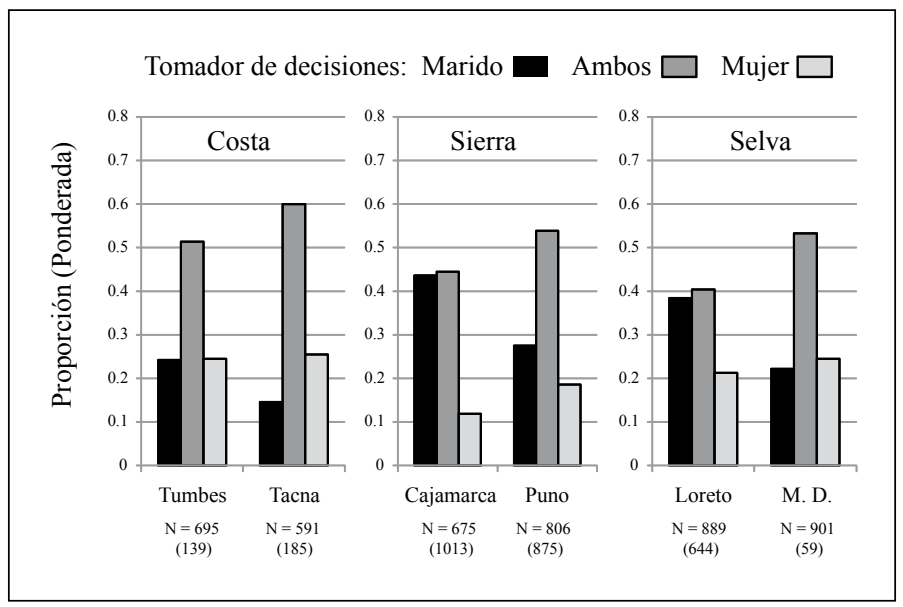

Figura 1. Proporción en que la informante, su marido, o ambos toman decisiones sobre grandes compras en las regiones políticas más septentrionales y meridionales del Perú, por región natural. 


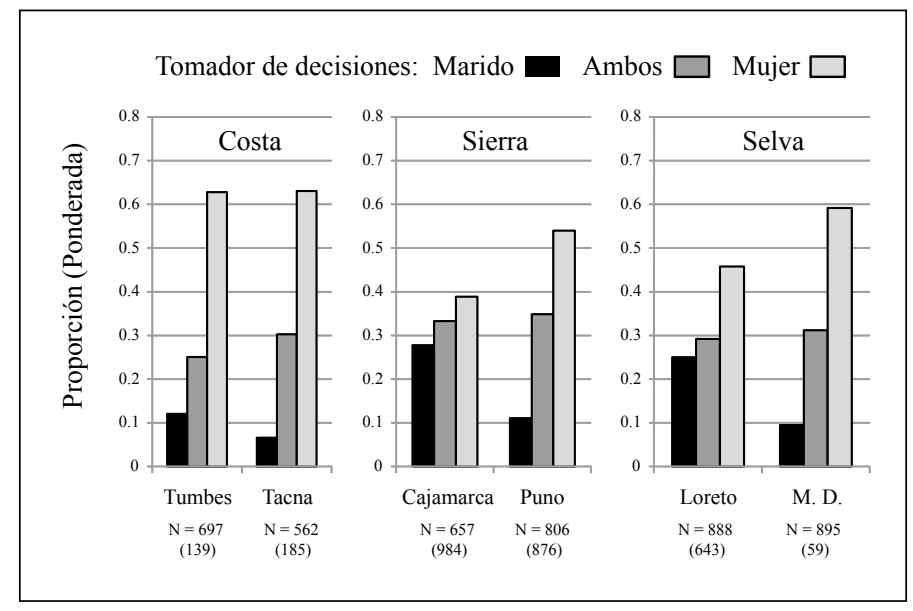

Figura 2. Proporción en que la informante, su marido, o ambos toman decisiones sobre compras diarias en las regiones políticas más septentrionales y meridionales del Perú, por región natural.

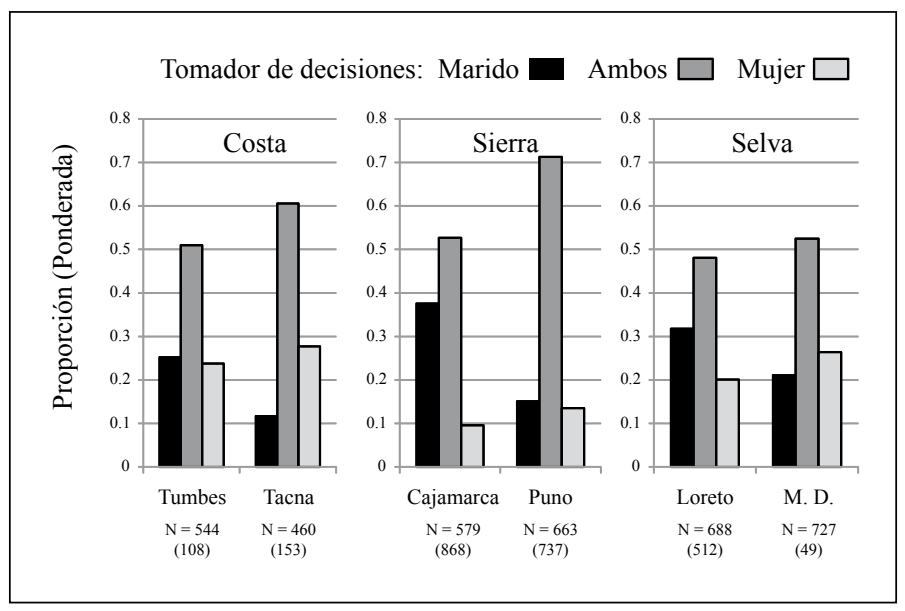

Figura 3. Proporción en que la informante, su marido, o ambos toman decisiones sobre los ingresos del marido en las regiones políticas más septentrionales y meridionales del Perú, por región natural. 
Los resultados para el ítem sobre grandes compras del hogar muestran que el poder del marido es mayor en el norte que en el sur en las tres regiones mientras que lo contrario ocurre con las decisiones conjuntas y las autónomas de la mujer. Lo mismo se encuentra frente a compras menudas; obsérvese que en este caso las decisiones autónomas de la mujer superan a las conjuntas. La relación latitud-decisiones recurre respecto a la variable sobre el uso de los ingresos del marido. Aquí se nota una importante pérdida de casos; la disminución del número de respuestas, comparada a la de los ítems sobre grandes compras o compras para necesidades diarias, probablemente expresa silencios de la mujer en casos de ausencia de ingresos del marido. En las tres áreas decisionales el poder de la mujer está maximizado en la costa y minimizado en la sierra. Los resultados sobre compras grandes e ingresos del marido se diferencian de los pertinentes a compras menudas, sugiriendo que las decisiones importantes favorecen una mayor presencia de elecciones conjuntas, mientras que en las decisiones secundarias la mujer adquiere mayor autonomía. Transcendiendo estas circunstancias, los resultados apoyan en cada caso la hipótesis de un mayor poder doméstico de la mujer sureña.

\section{Relaciones entre variables}

La tabla 1 presenta las relaciones entre latitud y las variables sociodemográficas en las nueve sub-muestras.

Considerando que la probabilidad de recurrencia del mismo signo de la correlación por mero azar al menos en ocho de las nueve submuestras sería menor que .04 (dos colas) según el test binomial, se puede concluir razonablemente que, de norte a sur, aumenta la riqueza del hogar en el Perú, disminuye la frecuencia del castellano como lengua materna, disminuye la diferencia de edad de la mujer con la del marido, y aumentan el nivel de educación de la mujer, la frecuencia del trabajo rentado de ella, su visión de TV, y su lectura de periódicos. El análisis de consistencia interna indicó la combinabilidad de la riqueza del hogar, educación de la mujer, y su visión de TV y lectura de periódicos en un indicador sumatorio $(\alpha=.82)$ al que se le puede llamar 


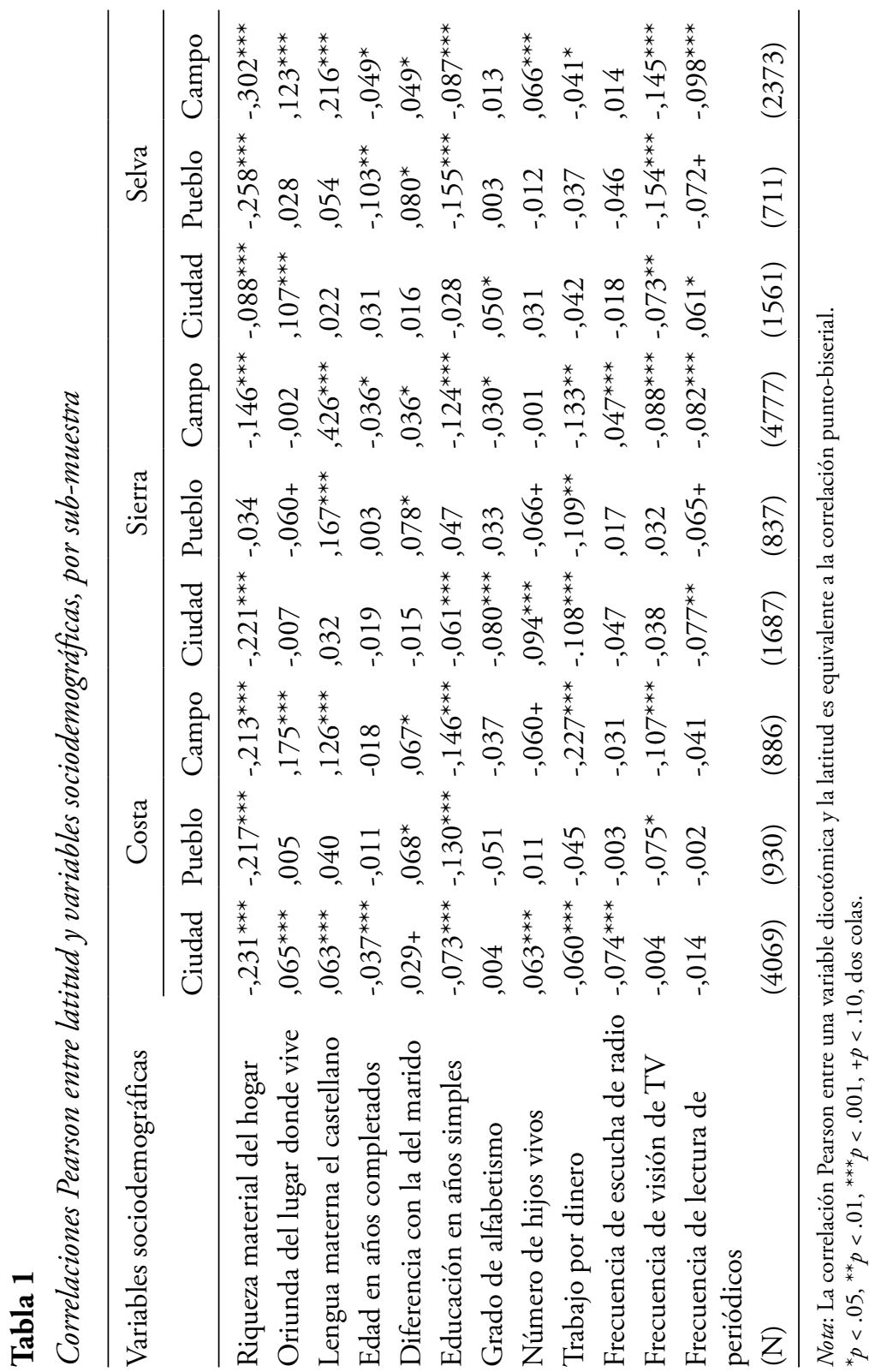


poder material/informacional. Las otras variables correlacionadas con la meridionalidad no son combinables en una suma.

La tabla 2 presenta los resultados de las regresiones de cada área decisional sobre la latitud y las variables sociodemográficas sobrevivientes. Los resultados que involucran a la latitud y las decisiones de la mujer son notablemente coherentes; de no existir relación entre latitud y decisiones, los coeficientes fluctuarían por encima y por debajo de 1; en cambio, casi todos son negativos (menores que 1) y la mayoría de ellos alcanzan o bordean la significación estadística. Los efectos significativos son ingentes.

Aún en el caso de las ciudades de la costa, que presentan efectos relativamente débiles, la probabilidad de una decisión conjunta en las grandes compras, comparada con la probabilidad de una decisión hecha solo por el marido, cae en 3,5\% por cada grado de latitud que acerca a la mujer a la línea ecuatorial. Es decir, la probabilidad contingente (odds) se incrementaría en casi 5\% de Tumbes a Piura, en 24\% de Piura a Lima, y en $51 \%$ de Tumbes a Tacna. Los mayores tamaños de muestra contribuyen a dar significación estadística a la relación latitud-decisiones en las ciudades de la costa y el campo de la sierra y la selva, pero no explican las marcadas ausencias de ella en ciudades de la sierra, pues más resultados significativos se observan en los pueblos de cualquier región pese a su menor número de casos. Globalmente, la relación de la latitud con las decisiones femeninas emergió más fuerte en los pueblos y campo del Perú que en las ciudades.

Considerando que el eje norte-sur de decisiones domésticas podría manifestarse de manera diferente en diferentes ámbitos, conviene evaluar los resultados por área decisional y tipo de decisión. Aquí al autor solo le interesaron los coeficientes significativos, pues los otros tienen errores estándar demasiado grandes como para tenerlos en cuenta. Con pocas excepciones, los resultados son similares para decisiones conjuntas o autónomas, no así a través de áreas. La relación latitud-decisiones es más consistente en el área del uso del ingreso del marido que en las de compras pese al menor número de casos. En efecto, se observa en la tabla 2 para el ingreso del marido, pero no para las compras, relaciones 
significativas de la latitud con las decisiones autónomas, conjuntas, o ambas en las nueve sub-muestras. En cambio, es llamativa la falta de relaciones significativas para cualquier tipo de decisión en cualquier área de compras en las ciudades de la sierra. De las variables sociodemográficas, el determinante más potente y consistente del poder doméstico de la mujer es su poder material/informacional. El castellano como lengua materna también presenta efectos positivos; su relevancia se observa a través de áreas decisionales en los pueblos y campo de sierra y selva. Los efectos de la diferencia de edad son negativos y más puntuales. El trabajo rentado de la mujer afecta más las decisiones de compras que las de uso del ingreso del marido.

\section{Comentarios}

Antes de considerar el significado de los resultados para la hipótesis de León (1984) hay que discutir las implicancias de las correcciones de región natural que fue preciso hacer para enmendar errores en la base de datos, evaluar la consistencia de los hallazgos con la literatura empírica, y juzgar las limitaciones de la estrategia analítica empleada.

Es probable que los errores observados hayan debido su existencia a la naturaleza de la variable costa-sierra-selva, que, siendo idiosincrática del Perú, Ecuador y Colombia, puede haber quedado excluida de la fiscalización por parte de los algoritmos estándar de limpieza de datos de DHS. Parece improbable que haya habido errores de codificación o transcripción en las otras variables usadas en este estudio; la de etnicidad también es de carácter local, pero de ninguna manera tan difícil de codificar como la región natural. De otro lado, si se asumiera que todas las variables incluidas en el estudio contenían errores de codificación o transcripción como aquellos observados para la región natural, no hay razón para suponer que afectaran a más del 1\% de los casos y de forma no-aleatoria. Es decir, habrían hecho algo más difícil la confirmación de la hipótesis, pero no introducido sesgos. En cualquier caso, poco habría cambiado el patrón de resultados de no haberse introducido las correcciones. 


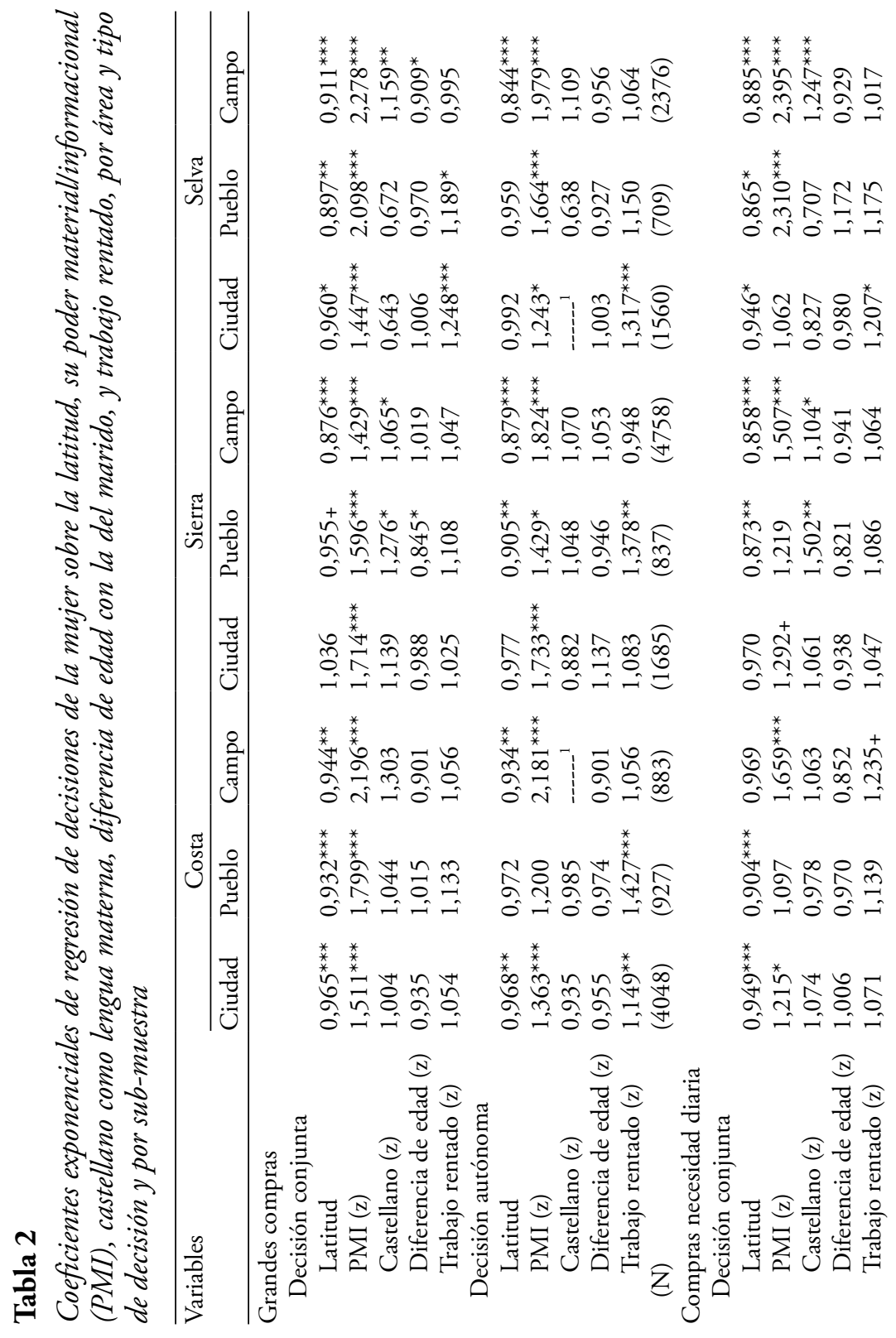




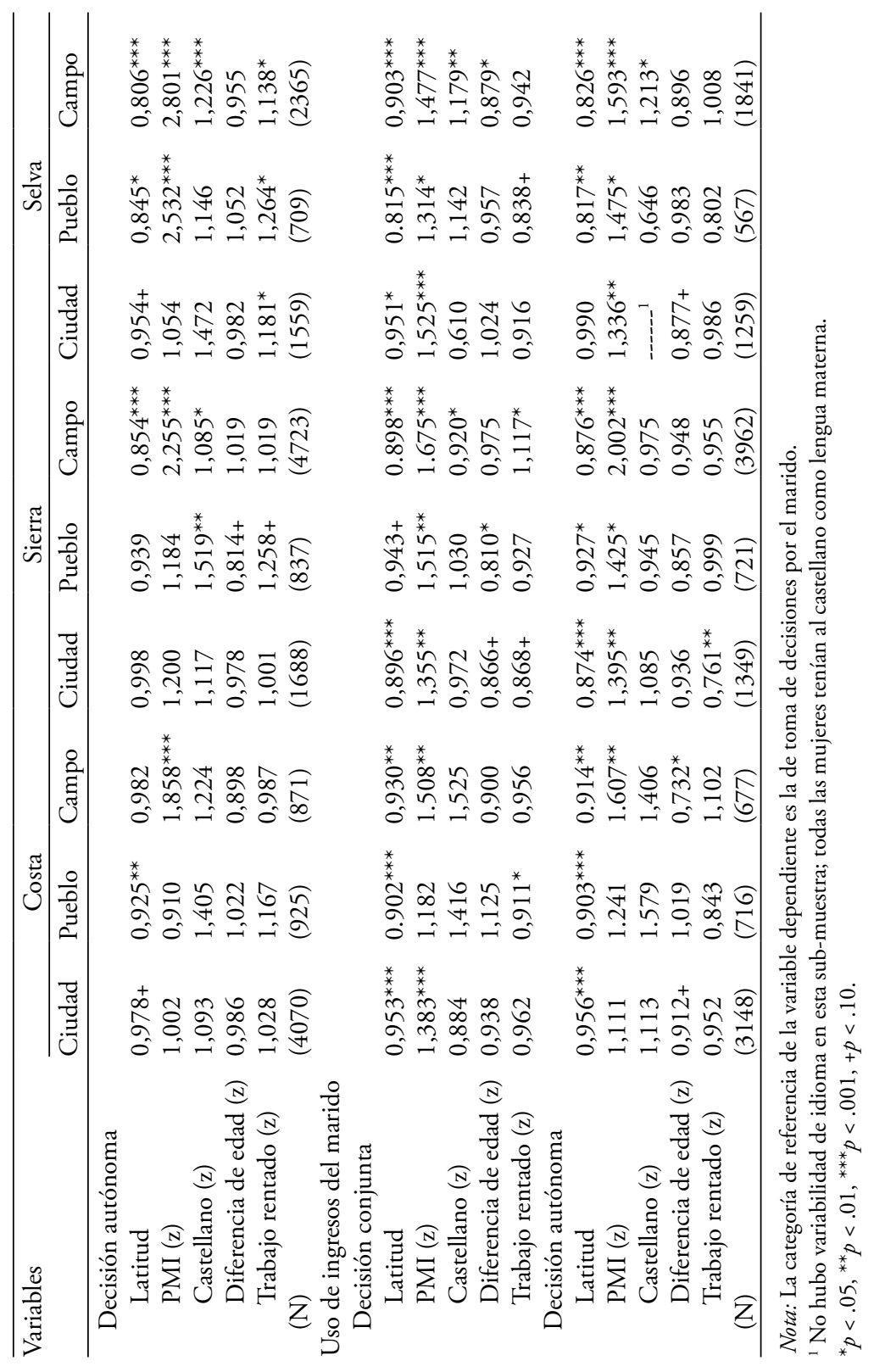


El significativo crecimiento de norte a sur de la riqueza material del hogar puede parecer el más sorprendente de los resultados presentados en este artículo, pues existe la percepción de que el sur del Perú contiene la mayor pobreza del país. Esta percepción, sin embargo, es errónea, como se constata en el mapa de la pobreza extrema del Perú; por ejemplo, Tumbes está en el quintil 3, Tacna en el 4, Cajamarca en el 1, Puno en el 2, Loreto en el 1 y Madre de Dios en el 3 (FONCODES, 2006). Tampoco contradice la literatura empírica el hallazgo de un trabajo femenino rentado que crece de norte a sur. A falta de mejor información, se puede calcular a partir de los datos del Censo de 2007 (INEI, 2008) el número de mujeres económicamente activas (Cuadro 3.9) relativo al número de mujeres en edad fértil (Cuadro 1.40); se encontrará, por ejemplo, que alcanza $40 \%$ en Tumbes versus $66 \%$ en Tacna; $33 \%$ en Cajamarca versus 59\% en Puno; y 45\% en Loreto versus 54\% en Madre de Dios. Los otros hallazgos del estudio son consistentes con estos y/o con expectativas corrientes en las ciencias sociales peruanas.

Los dos enfoques analíticos empleados fueron imperfectos. El de los departamentos extremos produce información sencilla y nada ambigua pero ignora al resto del Perú y las posibles variables contaminantes (e. g. Tacna cuenta con sierra, Tumbes no). El enfoque de la relación entre variables tiene las ventajas de la integralidad y la concepción multivariada y la desventaja de las dudas que sus resultados pueden dejar debido al desbalance de representatividad de los casos. Esto se debe a que el muestreo de la Encuesta Continua 2004-2008 se hizo por departamentos mientras que la segmentación usada aquí consideró el cruce de región natural por tipo de lugar de residencia. Por ejemplo, Lima Metropolitana está sub-representada en la sub-muestra de ciudades de la costa, el área urbana de Madre de Dios está sobre-representada entre los pueblos de la selva, y así sucesivamente.

No obstante todo ello, proviniendo de enfoques complementarios, los resultados dejan escasa incertidumbre sobre la estrecha relación existente entre latitud y control de los ingresos del marido por la mujer en el Perú: el poder de ella en esta área es mayor en el sur que en el norte según el primer enfoque y se incrementa sistemáticamente de norte a 
sur según el segundo. Para calificar de espuria la última relación habría que demostrar la presencia de un sesgo común a las nueve sub-muestras. La conjetura de León (1984) ha generado, pues, el descubrimiento de un hecho empírico sólido. La segunda conclusión establece que la relación de la latitud con el poder de la mujer en el área de las compras hogareñas es menos consistente que en la del uso del ingreso del marido, pues se confirma con el enfoque de departamentos extremos pero bajo el de relaciones entre variables presenta la excepción de las ciudades de sierra. Esto podría deberse a algún sesgo específico de representatividad en la sub-muestra de ciudades de la sierra, a características sociales que diferencian a estas ciudades de las de las otras regiones, y/o a ciertas implicancias de las áreas decisionales. Que una mujer sea autónoma en las compras de necesidad diaria y que otra tome por su cuenta las decisiones sobre cómo debe usarse el ingreso del marido dicen cosas diferentes sobre las relaciones de poder en la pareja.

Los hechos empíricos constatados cubren el constructo "autonomía de la mujer versus dependencia del marido" (León, 1984) de manera limitada al referirse solo a las decisiones económicas del hogar cuando aquél tiene un ámbito considerablemente más vasto, que las trasciende. Al mismo tiempo, conducen a un terreno conceptual de mayor especificidad que el tocado por los estudios previos. Bajo el enfoque por departamentos se midió el poder decisional de la mujer en bruto, es decir, sin tener en cuenta las fuentes de su poder, pero bajo el enfoque de las relaciones entre variables se ajustó la relación latitud-decisiones considerando los efectos de las variables sociodemográficas usadas. Es decir, cada coeficiente exponencial de regresión del comportamiento decisorio de la mujer sobre la latitud fue calculado manteniendo constantes los efectos de su poder material/informacional, además de los de las otras variables consideradas. El poder de la mujer en el área doméstica tiene diversas fuentes. Por un lado están las normas sociales que amplían o restringen el rango de comportamientos permisibles según el género de la persona (Kabeer, 2001; Mason, 1998; Sen, 1999). Este factor estuvo representado en el presente estudio por la variable que distinguió las etnias autóctonas de la que tiene al castellano como lengua 
materna. La literatura también demuestra en el plano internacional que la edad de la mujer, su nivel educativo y su trabajo por dinero fortalecen su poder doméstico, y que la diferencia de edad con la del marido debilita sus decisiones autónomas, aunque no las conjuntas (Kishor \& Subaiya, 2008). Comoquiera que estas variables, con excepción de la edad, que no correlacionó con latitud con la debida consistencia, fueron mantenidas constantes en las regresiones del estudio reportado aquí, se debe concluir que el control económico del hogar por la mujer que se halló relacionado con la latitud tiene una fuente distinta de poder.

Este autor imagina que tal poder residual de la mujer, es decir, no explicado por las otras variables, proviene de un rasgo de personalidad, específicamente, la asertividad, un aspecto de la extraversión (Eysenck \& Eysenck, 1985; Goldberg, 1973). Pues, ¿qué otra cosa podría determinar con más fuerza el poder doméstico de la mujer aparte de las normas sociales de comportamiento que hay en su entorno, su poder material/informacional, la diferencia de edad con la del marido, y el que trabaje y gane dinero? Aunque tangencialmente, la asertividad ha sido estudiada a través de sociedades y culturas. Así, está demostrado que el hombre es más asertivo que la mujer, pese a que la variabilidad al interior de los géneros puede ser mayor que entre ellos (Costa, Terracciano \& McCrae, 2001). Lo que habría que preguntar es si la asertividad del hombre peruano también crece de norte a sur o si son las diferencias de género en asertividad las que disminuyen de norte a sur en el Perú.

Con el foco en las diferencias de género en personalidad viene una ampliación del horizonte conceptual. Las principales perspectivas desde las que estas se han encarado contrastan la asertividad con el amor o calidez. Bem (1974) desarrolló escalas ortogonales de masculinidad-femineidad que son esencialmente mediciones de dominio y amor; poder y amor son también los ejes del circumplejo interpersonal de Wiggins (1979); y en la perspectiva de los cinco grandes factores de personalidad bajo el enfoque de medición del NEO-PI-R (Costa \& McCrae, 1992), las facetas relevantes son asertividad y calidez. La figura 4 plantea una hipótesis sobre las relaciones entre la latitud y el circumplejo. 


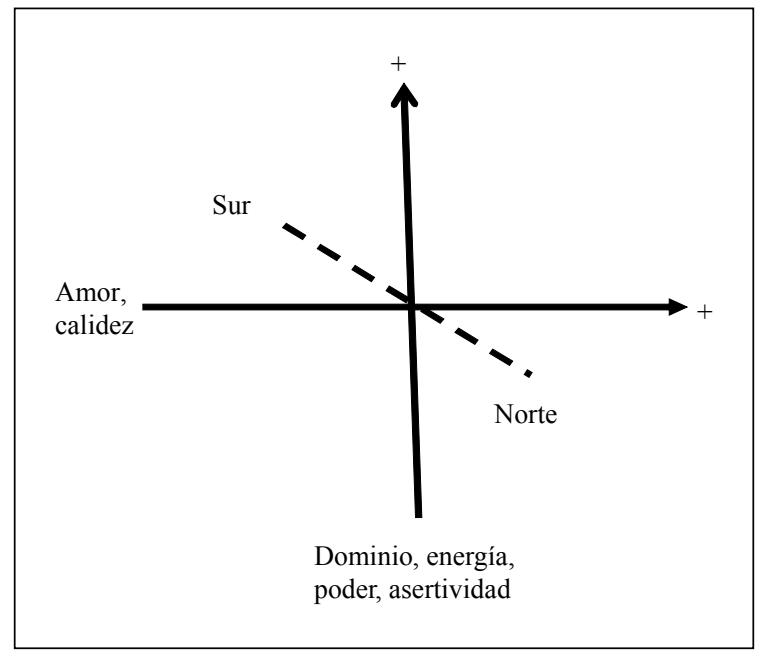

Figura 4. Relaciones hipotéticas entre la latitud sur y el circumplejo interpersonal de Wiggins (1979).

León (1986) propuso una re-interpretación del eje fecundatorio norte-sur que está relacionada con la dimensión del amor o calidez, anque no reparó en ello en su momento. La propuesta surgió en el contexto de un análisis de respuestas de mujeres solteras diseñado en un intento de entender por qué las mujeres norteñas, muy fértiles e ignorantes de anticonceptivos en los años 1960, habían mejorado sus conocimientos de planificación familiar con mayor efectividad que las de otras regiones y controlaban su fecundidad igual o mejor que ellas en los 1970s, pese a lo cual seguían diciendo que deseaban tener hijos en mayor medida. Esto se explicaría porque la sexualidad sería más central en la vida de la mujer a medida que se acerca a la línea ecuatorial.

Para apuntalar la hipótesis, León (1986) se refirió a los restos arqueológicos pre-hispánicos de contenido sexual explícito que fueron analizados por Kauffmann-Doig (1979), donde se aprecia claramente una presencia dominante de culturas del norte (Vicús, Moche) y bastante menor de culturas del centro (Chancay, Nazca) y especialmente del sur (Tiahuanaco, Inca). Si este eje norte-sur erótico no expresa simplemente 
una mayor producción de cerámicas de todo tipo en el norte, los datos de Kauffmann-Doig (1979) sugieren que un culto a la sexualidad existió en el antiguo Perú, decreciendo con la distancia al Ecuador. Sexualidad y amor son parte de la libido freudiana. La segunda hipótesis explicativa de León podría, pues, verificarse midiendo el interés sexual de la mujer y/o el rasgo de amor/calidez a lo largo del eje geográfico norte-sur del Perú. Más completo, por supuesto, sería abordar ambos, asertividad y calidez, en hombres y mujeres. Las investigaciones, evidentemente, tendrían que trascender las encuestas DHS, pues sería necesario emplear mediciones específicas de estos rasgos de personalidad.

\section{Referencias}

Al Riyami, A., Afifi, M. \& Mabry, R. M. (2004). Women's autonomy, education, and employment in Oman and their influence on contraceptive use. Reproductive Health Matters, 12, 144-154.

Alcántara, E. (1983). Conocimiento de métodos anticonceptivos. En Instituto Nacional de Estadística, Aspectos demográficos y prevalencia de anticonceptivos: resultados de la Primera Encuesta Nacional de Prevalencia de Anticonceptivos. Lima: Autor.

Baumrind, D. (1985). Familial antecedents of adolescent drug use: A developmental perspective. En C. L. Jones \& R. J. Battjes (Eds.), Etiology of drug abuse: Implications for prevention. Rockville, MD: National Institute on Drug Abuse. (NIDA Research Monograph No. 56.)

Bem, S. L. (1974). The measurement of psychological androgyny. Journal of Consulting and Clinical Psychology, 42, 115-162.

Blanc, A. K. (2001). The effect of power in sexual relationships on sexual and reproductive health: An examination of the evidence. Studies in Family Planning, 32, 189-213.

Blum, R. H., Henry, W. E. \& Sanford, N. (Eds.). (1972). The dream sellers. San Francisco, CA: Jossey-Bass. 
Braucht, G. N., Brakarsh, D., Follingstad, D. \& Berry, K. L. (1973). Deviant drug use in adolescence: A review of psychosocial correlates. Psychological Bulletin, 79, 92-106.

Brook, J. S., Linkoff, I. F. \& Whitman, M. (1980). Initiation into adolescent marijuana use. Journal of Genetic Psychology, 137, 133-142.

Casique, I. (2003). Uso de anticonceptivos en México: ¿Qué diferencia hacen el poder de decisión y la autonomía femenina? Papeles de Población, 35, 209-233. Recuperado de http://redalyc.uaemex. mex/pdf/112/11203510.pdf

Chavoshi, M. H., Abbasi-Shavazi, M. J. \& McDonald, P. (Septiembre, 2004). Women's autonomy and reproductive behavior in Iran. Trabajo presentado en la $12^{\text {th }}$ Biennial Conference of the Australian Population, Camberra. Recuperado de http://www.apa.org.au/ upload/2004-5E_Chavoshi.pdf

Costa Jr., P. T. \& McCrae, R. R. (1992). Revised NEO Personality Inventory (NEO-PI-R) and NEO Five Factor Inventory (NEOFFI) professional manual. Odessa, FL: Psychological Assessment Resources.

Costa Jr., P. T., Terracciano, A. \& McCrae, R. R. (2001). Gender differences in personality traits across cultures: Robust and surprising findings. Journal of Personality and Social Psychology, 81, 322-331.

Cronbach, L. J. (1951). Coefficient alpha and the internal structure of tests. Psychometrika, 16, 297-334.

DHS. (2010). Bases de datos de Peru Continuous 2004-2008 Demographic and Health Survey. (Proporcionadas por Demographic and Health Surveys para el proyecto Women's decision-making power and use of contraception.)

Eysenck, H. J. \& Eysenck, M. W. (1985). Personality and individual differences: A natural science approach. Nueva York: Plenum.

Feldman, B. S., Zaslavsky, A. M., Ezzati, M., Peterson, K. E. \& Mitchell, M. (2009). Contraceptive use, birth spacing, and autonomy: An analysis of the Oportunidades program in rural Mexico. Studies in Family Planning, 40, 51-62.

FONCODES. (2006). Mapa de pobreza 2006. Lima: Autor. 
Goldberg, L. R. (1993). The structure of phenotipic personality traits. American Psychologist, 48, 26-34.

INEI. (1983). Aspectos demográficos y prevalencia de anticonceptivos: resultados de la Primera Encuesta Nacional de Prevalencia de Anticonceptivos. Lima: Autor.

INEI, UNFPA \& PNUD. (2008). Censos Nacionales 2007: XI de Población y VI de Vivienda (2da. ed.). Lima: Autor.

INEI \& ORC Macro. (2010). Perú: Encuesta Demográfica y de Salud Familiar - ENDES Continua, 2009. Lima: Autor.

Jejeebhoy, S. J. (2002). Convergence and divergence in spouses' perspectives on women's autonomy in rural India. Studies in Family Planning, 33, 299-308.

Jutkowitz, J. M., Arellano, R., Castro de la Mata, R., Davis, P. B., Elinson, J., Jerí, F. R. et al. (1986). Drug use and abuse in Peru: An epidemiological investigation of drugs in urban Peru. Lima: Development Associates.

Kabeer, N. (2001). Reflections on the measurement of women's empowerment. En SIDA Studies No. 3, Discussing women's empowerment: Theory and practice. Estocolmo: Novum Grafiska AB.

Kandel, D. B. (1982). Epidemiological and psychological perspectives on adolescent drug use. Journal of American Academic and Clinical Psychiatry, 21, 328-347.

Kandel, D. B., Kessler, R. \& Margulies, R. (1978). Antecedents of adolescents initiation into stages of drug use: A developmental analysis. En D. B. Kandel (Ed.), Longitudinal research in drug use: Empirical findings and methodological issues. Washington, DC: Hemisphere-Wiley.

Kaplan, H. B. (1982). Self-attitudes and deviant behavior: New directions for theory and research. Youth and Society, 14, 185-211.

Kauffmann-Doig, F. (1979). Sexual behavior in ancient Peru. Lima: Kompaktos.

Kim, S. (1979). An evaluation of Ombudsman Primary Prevention Program on student drug abuse. Charlotte, NC: Charlotte Drug Education Center. 
Kishor, S. \& Gupta, K. (2009). Gender equality and women's empowerment in India. Mumbai: International Institute for Population Sciences.

Kishor, S. \& Subaiya, L. (2008). Understanding women's empowerment: A comparative analysis of Demographic and Health Surveys data. DHS Comparative Studies No. 20.

León, F. R. (1984). El eje fecundatorio norte-sur del Perú: una interpretación psicológica. Revista de Psicología, 2, 95-111.

León, F. R. (1986). Factores psicosociales, psicoeconómicos, y psicosexuales en el eje fecundatorio norte-sur del Perú. En F. R. León (Ed.), Psicología y realidad peruana: el aporte objetivo. Lima: Mosca Azul.

León, F. R. (1987). El eje psicoactivo norte-sur del Perú. Psicoactiva, 1, 3-13.

León, F. R. (2011). Does professed religion moderate the relationship between women's domestic power and contraceptive use in India? Open Family Studies Journal, 4, 1-8.

Malhotra, A., Schuler, S. R. \& Boender, C. (2002). Measuring women's empowerment as a variable in international development. Washington, DC: World Bank's Social Development Group.

Mason, K. O. (1998). Wive's economic decision-making power in the family: Five Asian countries. En K. O. Mason (Ed.), The changing family in comparative perspective: Asia and the United States. Honolulu: East-West Center.

Morales, T. (1983). Intenciones reproductivas. En Instituto Nacional de Estadística e Informática, Aspectos demográficos y prevalencia de anticonceptivos: resultados de la Primera Encuesta Nacional de Prevalencia de Anticonceptivos. Lima: Autor.

Moursund, A. \& Kravdal, O. (2003). Individual and community effects of women's education and autonomy on contraceptive use in India. Population Studies, 57, 285-301.

Mumtaz, Z., Slaymaker, E. \& Salway, S. (2005). Condom use in Uganda and Zimbabwe: Exploring the influence of gendered access to resources and couple-level dynamics. En S. Kishor (Ed.), A focus 
on gender: Collected papers using DHS data. Calverton, MD: ORC Macro.

Penning, M. \& Barnes, G. E. (1982). Adolescent marijuana use: A review. International Journal of the Addictions, 17, 749-791.

Pulgar Vidal, J. (1938). Las ocho regiones naturales del Perú (Tesis inédita). Universidad Nacional Mayor de San Marcos, Lima, Perú.

Roy, T. K. \& Niranjan, S. (2004). Indicators of women's empowerment in India. Asia-Pacific Population Journal, 19, 23-40.

Saleem, S. \& Bobak, M. (2005). Women's autonomy, education and contraception use in Pakistan: A national study. Reproductive Health, 2, 8.

Sathar, Z., Callum, C. \& Jejeebhoy, S. (Agosto, 2001). Gender, region, religion and reproductive behaviour in India and Pakistan. Trabajo presentado en la XXIV IUSSP General Population Conference, Salvador, Brasil. Recuperado de http://www.iussp.org/Brazil2001/ s80/S87_02_sathar.pdf

Sen, A. (1999). Development as freedom. Oxford: Oxford University Press.

SPSS. (2008). SPSS for Windows, Rel. 17.0.1. Chicago: Autor.

Wiggins, J. S. (1979). A psychological taxonomy of trait descriptive terms: The interpersonal domain. Journal of Personality and Social Psychology, 37, 395-412.

Woldemicael, G. (2009). Women's autonomy and reproductive preferences in Eritrea. Journal of Biosocial Science, 41, 161-181.

Recibido: 2 de marzo, 2011 Aceptado: 30 de julio, 2011 